PROCEEDINGS OF THE

AMERICAN MATHEMATICAL SOCIETY

Volume 131, Number 6, Pages 1711-1716

S 0002-9939(03)06893-X

Article electronically published on January 15, 2003

\title{
AN ALGEBRAIC PROPERTY OF JOININGS
}

\author{
YOUNG-HO AHN AND MARIUSZ LEMAŃCZYK
}

(Communicated by Michael Handel)

\begin{abstract}
We show that an ergodic automorphism is semisimple if and only if the set of ergodic self-joinings is a subsemigroup of the semigroup of selfjoinings.
\end{abstract}

\section{INTRODUCTION}

Assume that $T$ is an ergodic automorphism of a probability standard Borel space $(X, \mathcal{B}, \mu)$. By $J(T)$ we denote the set of all self-joinings of $T$ that are all $T \times T$ invariant measures defined on $(X \times X, \mathcal{B} \otimes \mathcal{B})$, both of whose natural projections are equal to $\mu$. On the set $J(T)$ there is a natural structure of a semitopological compact affine semigroup (see the next section for this and some further basic notions and results). By $J^{e}(T)$ we denote the set of ergodic members of $J(T)$.

In [3], A. del Junco, M.K. Mentzen and the second author introduced a notion of semisimplicity. We say that $T$ is semisimple if for any $\lambda \in J^{e}(T)$ the automorphism $(T \times T, \lambda)$ is relatively weakly mixing over $T$ ( $T$ is given by the projection on the first coordinate). The notion of semisimplicity generalized the notion of minimal self-joinings [7] and of simplicity [4, 9]. Moreover, some Gaussian automorphisms turned out to be semisimple (see [5]). It follows from basic properties of relative products that $J^{e}(T)$ is stable under composition whenever $T$ is semisimple. The aim of this note is to prove that the converse also holds.

Theorem 1. Let $T$ be an ergodic automorphism of $(X, \mathcal{B}, \mu)$. Then $T$ is semisimple if and only if the set of ergodic self-joinings is a subsemigroup of $J(T)$.

This note was written during the first author's stay at the Nicholas Copernicus University in the academic year 2000/2001.

\section{Notation AND BASIC RESUlts}

Suppose that $\pi:(Z, \mathcal{D}, \rho) \rightarrow(Y, \mathcal{C}, \eta)$ is a homomorphism of two standard probability spaces. Given $f \in L^{1}(Z, \rho)$, by $E(f \mid Y)$ or $E^{\eta}(f \mid Y)$ we denote the conditional expectation of $f$ with respect to $Y$, i.e. the function in $L^{1}(Y, \eta)$ given by

Received by the editors July 14, 2001.

2000 Mathematics Subject Classification. Primary 28D05, 37A05.

Key words and phrases. Joining, composition of joinings, semisimple, compact extension, relatively independent product, relative weak mixing.

The first author was supported by the KOSEF postdoctoral fellowship program and the Nicholas Copernicus University.

The second author's research was partly supported by KBN grant P03A 02721 (2001).

(C)2003 American Mathematical Society 
$E\left(f \mid \pi^{-1}(\mathcal{C})\right) \circ \pi^{-1}$. If

$$
\rho=\int_{Y} \rho_{y} d \eta(y)
$$

denotes the disintegration of $\rho$ over $\eta$, then $E(\cdot \mid Y)(y)=\rho_{y}(\cdot)$ for a.a. $y \in Y$ (see [2], Th. 5.8). If $\pi^{\prime}:\left(Z^{\prime}, \mathcal{D}^{\prime}, \rho^{\prime}\right) \rightarrow(Y, \mathcal{C}, \eta)$ is another homomorphism and $\rho^{\prime}=\int_{Y} \rho_{y}^{\prime} d \eta(y)$, then the measure

$$
\rho \otimes_{Y} \rho^{\prime}=\int_{Y} \rho_{y} \otimes \rho_{y}^{\prime} d \eta(y)
$$

defined on $\mathcal{D} \otimes \mathcal{D}^{\prime}$ is called the relative product of $\rho$ and $\rho^{\prime}$ over $(Y, \eta)$ (see [2], Chapter $5, \S 5)$. The resulting space will be denoted $\left(Z \times_{Y} Z^{\prime}, \rho \otimes_{Y} \rho^{\prime}\right)$. In what follows we will need the following.

Lemma 1. Consider a sequence of homomorphisms $(Z, \mathcal{D}, \rho) \rightarrow(Y, \mathcal{C}, \eta) \rightarrow(X, \mathcal{B}, \mu)$. Whenever $f, g \in L^{2}(Z, \rho)$, then $E\left(f \otimes g \mid Y \times_{X} Y\right)=E(f \mid Y) \otimes E(g \mid Y), \eta \otimes_{X} \eta$ a.s.

Proof. Let $\rho=\int_{Y} \rho_{y} d \eta(y), \rho=\int_{X} \tilde{\rho}_{x} d \mu(x)$ and $\eta=\int_{X} \eta_{x} d \mu(x)$ stand for the relevant disintegrations. We have

$$
\begin{gathered}
\rho(A)=\int_{Z} \chi_{A} d \rho=\int_{Y} E\left(\chi_{A} \mid Y\right)(y) d \eta(y) \\
=\int_{X}\left(\int_{Y} E\left(\chi_{A} \mid Y\right)(y) d \eta_{x}(y)\right) d \mu(x)=\int_{X}\left(\int_{Y} \rho_{y}(A) d \eta_{x}(y)\right) d \mu(x) ;
\end{gathered}
$$

thus

Hence

$$
\tilde{\rho_{x}}=\int_{Y} \rho_{y} d \eta_{x}(y)
$$

It follows that

$$
\tilde{\rho_{x}} \otimes \tilde{\rho_{x}}=\int_{Y \times Y} \rho_{y} \otimes \rho_{y^{\prime}} d \eta_{x} \otimes \eta_{x}\left(y, y^{\prime}\right) .
$$

$$
\begin{gathered}
\rho \otimes_{X} \rho=\int_{X} \tilde{\rho_{x}} \otimes \tilde{\rho_{x}} d \mu(x) \\
=\int_{X}\left(\int_{Y \times Y} \rho_{y} \otimes \rho_{y^{\prime}} d \eta_{x} \otimes \eta_{x}\left(y, y^{\prime}\right)\right) d \mu(x)=\int_{Y \times Y} \rho_{y} \otimes \rho_{y^{\prime}} d \eta \otimes X \eta\left(y, y^{\prime}\right) .
\end{gathered}
$$

Hence, if $f, g \in L^{2}(Z, \rho)$, then

$$
\begin{gathered}
E\left(f \otimes g \mid Y \times_{X} Y\right)\left(y, y^{\prime}\right)=\int_{Y \times Y} f \otimes g d \rho_{y} \otimes \rho_{y^{\prime}} \\
=\int_{Y} f d \rho_{y} \int_{Y} g d \rho_{y^{\prime}}=E(f \mid Y)(y) \cdot E(g \mid Y)\left(y^{\prime}\right)
\end{gathered}
$$

and therefore $E\left(f \otimes g \mid Y \times_{X} Y\right)=E(f \mid Y) \otimes E(g \mid Y)$ a.s. with respect to $\eta \otimes_{X} \eta$.

Assume now that $T$ is an ergodic automorphism on a standard probability Borel space $(X, \mathcal{B}, \mu)$. To each element $\lambda \in J(T)$ we associate a Markov operator $\Phi_{\lambda}$ : $L^{2}\left(X_{1}, \mu_{1}\right) \rightarrow L^{2}\left(X_{2}, \mu_{2}\right)$ (where $\left(X_{i}, \mu_{i}\right)=(X, \mu)$, for $\left.i=1,2\right)$ given by

$$
\int_{X_{2}} \Phi_{\lambda}(f) \bar{g} d \mu_{2}=\int_{X_{1} \times X_{2}} f \bar{g} d \lambda .
$$

By Markov property we mean that $\Phi_{\lambda}$ is positive and $\Phi_{\lambda} 1=\Phi_{\lambda}^{*} 1=1$. We also have $\Phi_{\lambda} \circ T=T \circ \Phi_{\lambda}$. Moreover, for each $f \in L^{2}(X, \mu)$

$$
\left(\Phi_{\lambda} f\right)\left(x_{2}\right)=E^{\lambda}\left(f \mid X_{2}\right)\left(x_{2}\right) .
$$


Furthermore, each Markov operator on $L^{2}(X, \mu)$ that commutes with $T$ is necessarily of the form $\Phi_{\lambda}$ (see e.g. [5] or [8]). The latter observation introduces a semigroup law on $J(T)$ by the formula $\Phi_{\lambda_{2} \circ \lambda_{1}}=\Phi_{\lambda_{2}} \circ \Phi_{\lambda_{1}}$. Together with the weak topology and the natural simplex structure on $J(T)$ we obtain that $J(T)$ is a compact semitopological affine semigroup.

Suppose now $\lambda_{1}, \lambda_{2} \in J(T)$. We will treat $\lambda_{1}$ as defined on $X_{1} \times X_{2}$, while $\lambda_{2}$ is defined on $X_{2} \times X_{3}$. By $\lambda_{2}^{*}$ we mean the joining corresponding to $\Phi_{\lambda_{2}}^{*}$, that is, the self-joining given by

$$
\lambda_{2}^{*}\left(A_{2} \times A_{3}\right)=\lambda_{2}\left(A_{3} \times A_{2}\right) .
$$

Disintegrate $\lambda_{1}$ and $\lambda_{2}^{*}$ over the common factor $X_{2}$ :

$$
\lambda_{1}=\int_{X_{2}} \lambda_{1, x_{2}} d \mu_{2}\left(x_{2}\right), \quad \lambda_{2}^{*}=\int_{X_{2}} \lambda_{2, x_{2}}^{*} d \mu_{2}\left(x_{2}\right) .
$$

Consider the relative product of $\lambda_{1}$ and $\lambda_{2}^{*}$ over the common factor $X_{2}$ that is the measure defined on $X_{1} \times X_{2} \times X_{3}$ given by

$$
\lambda_{1} \otimes X_{2} \lambda_{2}^{*}=\int_{X_{2}} \lambda_{1, x_{2}} \otimes \lambda_{2, x_{2}}^{*} d \mu_{2}\left(x_{2}\right) .
$$

Take $f, g \in L^{2}(X, \mu)$. Using (1) we then have

$$
\begin{gathered}
\int_{X_{1} \times X_{3}} f\left(x_{1}\right) g\left(x_{3}\right) d \lambda_{1} \otimes X_{2} \lambda_{2}^{*}\left(x_{1}, x_{2}, x_{3}\right) \\
=\int_{X_{2}}\left(\int_{X_{1} \times X_{3}} f\left(x_{1}\right) g\left(x_{3}\right) d \lambda_{1, x_{2}} \otimes \lambda_{2, x_{2}}^{*}\left(x_{1}, x_{3}\right)\right) d \mu_{2}\left(x_{2}\right) \\
=\int_{X_{2}}\left(\Phi_{\lambda_{1}} f\right)\left(x_{2}\right)\left(\Phi_{\lambda_{2}^{*}} g\right)\left(x_{2}\right) d \mu_{2}\left(x_{2}\right)=\int_{X_{3}}\left(\Phi_{\lambda_{2}} \circ \Phi_{\lambda_{1}}\right)(f) g d \mu_{3} .
\end{gathered}
$$

We have shown the following:

$$
\lambda_{2} \circ \lambda_{1}=\left.\lambda_{1} \otimes_{X_{2}} \lambda_{2}^{*}\right|_{X_{1} \times X_{3}} .
$$

In particular, if $\lambda \otimes X_{2} \lambda^{*}$ is ergodic, then $\lambda \circ \lambda$ is ergodic and the key observation for the proof of Theorem 1 is that the converse is also true (see Proposition 1 below).

Let $T$ acting on $(X, \mathcal{B}, \mu)$ be a factor of an ergodic automorphism $S$ acting on $(Y, \mathcal{C}, \eta)$. Following 2] (see condition C5 on p. 132), we say that $S$ is a compact extension of $T$ if for each $0 \neq f \in L^{2}(Y, \eta)$ the limit of ergodic averages of $f \otimes \bar{f}$ for $S \times S$ acting on $\left(Y \times_{X} Y, \eta \otimes_{X} \eta\right)$ is also non-zero.

Remark 1. Usually a compact extension is defined in terms of relative eigenvectors (see [1, 10]). R. Zimmer proved in [10] that $S$ is a compact extension of $T$ if and only if $S$ is an isometric extension of $T$. Another proof of Zimmer's result follows easily from the joining characterization of isometric extensions given in [6].

Assume that $R$ acting on $(Z, \mathcal{D}, \rho)$ is an ergodic extension of $T$ acting on $(X, \mathcal{B}, \mu)$. Then (see [2], Chapter 6):

(A) there exists a biggest factor, called the relative Kronecker factor, $S$ acting on $(Y, \mathcal{C}, \eta)$ between $R$ and $T$ such that $S$ is a compact extension of $T$;

(B) the relative Kronecker factor $S$ is trivial (i.e. $S=T$ ) iff the relative product $R \times R$ on $\left(Z \times_{X} Z, \rho \otimes_{X} \rho\right)$ is ergodic (the latter condition means that $R$ is a relatively weakly mixing extension of $T)$. 
Finally, recall that an ergodic automorphism $T$ on $(X, \mathcal{B}, \mu)$ is called semisimple (3]) if for each $\lambda \in J^{e}(T)$, the relative product $\lambda \otimes_{X_{2}} \lambda^{*}$ is ergodic, that is (using (B)), $T \times T$ on $\left(X_{1} \times X_{2}, \lambda\right)$ is a relatively weakly mixing extension of $X_{2}$.

\section{Proof of Theorem 1}

We will need a lemma which is a simple consequence of the $L^{1}$-convergence in the pointwise ergodic theorem.

Lemma 2. Let $S$ be an automorphism on $(Y, \mathcal{C}, \eta)$. Denote by $\mathcal{I}$ the $\sigma$-algebra of $S$-invariant sets. Assume that $\mathcal{E} \subset \mathcal{C}$ is a factor of $S$. Then:

(i) If the action of $S$ on $\mathcal{E}$ is ergodic, then $E(f \mid \mathcal{I})=\int_{Y} f d \eta$ for each $f \in L^{1}(\mathcal{E})$.

(ii) If $f \in L^{1}(Y, \eta)$ and the sequence $\left(\frac{1}{n} \sum_{i=0}^{n-1} f \circ S^{i}\right)_{n \geq 1}$ converges to a constant $c\left(=\int_{Y} f d \eta\right)$, then $E(E(f \mid \mathcal{E}) \mid \mathcal{I})=c$.

Proof. (i) Since $f \in L^{1}(Y, \eta), \frac{1}{n} \sum_{i=0}^{n-1} f \circ S^{i}$ converges to $E(f \mid \mathcal{I})$ in $L^{1}(\mathcal{C})$ by the ergodic theorem. However $f$ is measurable with respect to $\mathcal{C}$ which is $S$-invariant. The result follows by the ergodicity of $S$ on $\mathcal{E}$.

(ii) Put $g=E(f \mid \mathcal{E})$. Then by the ergodic theorem, $\frac{1}{n} \sum_{i=0}^{n-1} g \circ S^{i}$ converges to $E(g \mid \mathcal{I})$ in $L^{1}(\mathcal{C})$ and hence in $L^{1}(\mathcal{E})$. Therefore for all $h \in L^{\infty}(\mathcal{E})$,

$$
\frac{1}{n} \sum_{i=0}^{n-1} \int f \circ S^{i} \cdot \bar{h} d \eta \rightarrow c \int \bar{h} d \eta
$$

We have

$$
\frac{1}{n} \sum_{i=0}^{n-1} \int f \circ S^{i} \cdot \bar{h} d \eta=\frac{1}{n} \sum_{i=0}^{n-1} \int E\left(f \circ S^{i} \mid \mathcal{E}\right) \cdot \bar{h} d \eta=\frac{1}{n} \sum_{i=0}^{n-1} \int E(f \mid \mathcal{E}) \circ S^{i} \cdot \bar{h} d \eta ;
$$

thus $\frac{1}{n} \sum_{i=0}^{n-1} g \circ S^{i}$ converges weakly to $c$ in $L^{1}(\mathcal{E})$. Hence $E(g \mid \mathcal{I})=c$.

The following lemma is a direct consequence of our definition of compact extension, the $L^{1}$-convergence of ergodic averages and the fact that $f \otimes \bar{f} \in L^{1}\left(Y \times_{X} Y\right)$ whenever $f \in L^{2}(Y, \eta)$.

Lemma 3. Let $S$ be an ergodic automorphism on $(Y, \mathcal{C}, \eta)$. Suppose that $S$ is a compact extension of $T$ acting on $(X, \mathcal{B}, \mu)$ and $f \in L^{2}(Y)$. Then $f=0$ if and only if $E(f \otimes \bar{f} \mid \mathcal{I})=0$ in the relative product $Y \times_{X} Y$.

The following result is of independent interest.

Proposition 1. Assume that $T$ is an ergodic automorphism of $(X, \mathcal{B}, \mu)$ and let $\lambda \in J^{e}(T)$. If $\lambda \circ \lambda$ is ergodic, then $\lambda \otimes_{X} \lambda^{*}$ is ergodic.

Proof. Given a real function $f \in L^{2}(X, \mu)$ put $f \otimes f\left(x_{1}, x_{2}, x_{3}\right)=f\left(x_{1}\right) f\left(x_{3}\right)$. We have $f \otimes f \in L^{1}\left(X_{1} \times X_{2} \times X_{3}, \lambda_{1} \otimes_{X_{2}} \lambda_{2}^{*}\right)$, where $\lambda_{1}=\lambda_{2}=\lambda$. If $\mathcal{I}$ denotes the $\sigma$-algebra of $T \times T \times T$-invariant sets in the relative product, then our ergodicity assumption on $\lambda \circ \lambda$ and (2) give rise to

$$
E(f \otimes f \mid \mathcal{I})=\int f \otimes f d \lambda \otimes_{X_{2}} \lambda^{*} .
$$


Let $(Y, \mathcal{C}, \eta)$ denote the relative Kronecker factor of $T \times T$ on $\left(X_{1} \times X_{2}, \lambda_{1}\right)$ over $X_{2}$ (see (A)). Then:

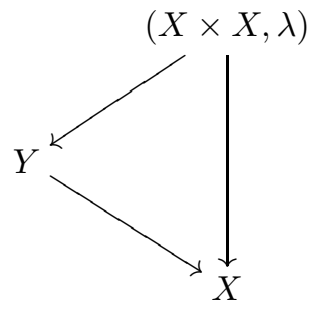

Fix a real function $f \in L^{2}(X, \mu)$. We will show that

$$
E\left(f\left(x_{1}\right) \mid Y\right)=E\left(f\left(x_{1}\right) \mid X_{2}\right) .
$$

Let $g=E\left(f\left(x_{1}\right) \mid Y\right)-E\left(f\left(x_{1}\right) \mid X_{2}\right)$. By Lemma 3 it is enough to prove that $E(g \otimes g \mid \mathcal{I})=0$ with respect to $\lambda_{1} \otimes_{X_{2}} \lambda_{2}^{*}$. We have

$$
\begin{gathered}
E(g \otimes g \mid \mathcal{I}) \\
=E\left(\left(E\left(f\left(x_{1}\right) \mid Y_{1}\right)-E\left(f\left(x_{1}\right) \mid X_{2}\right)\right) \otimes\left(E\left(f\left(x_{3}\right) \mid Y_{2}\right)-E\left(f\left(x_{3}\right) \mid X_{2}\right)\right) \mid \mathcal{I}\right) \\
=E\left(E\left(f\left(x_{1}\right) \mid Y_{1}\right) \otimes E\left(f\left(x_{3}\right) \mid Y_{2}\right) \mid \mathcal{I}\right)-E\left(E\left(f\left(x_{1}\right) \mid Y_{1}\right) \cdot E\left(f\left(x_{3}\right) \mid X_{2}\right) \mid \mathcal{I}\right) \\
-E\left(E\left(f\left(x_{1}\right) \mid X_{2}\right) \cdot E\left(f\left(x_{3}\right) \mid Y_{2}\right) \mid \mathcal{I}\right)+E\left(E\left(f\left(x_{1}\right) \mid X_{2}\right) \cdot E\left(f\left(x_{3}\right) \mid X_{2}\right) \mid \mathcal{I}\right) \\
=E\left(E\left(f\left(x_{1}\right) \mid Y_{1}\right) \otimes E\left(f\left(x_{3}\right) \mid Y_{2}\right) \mid \mathcal{I}\right)-\int_{Y_{1}} E\left(f\left(x_{1}\right) \mid Y_{1}\right) \cdot E\left(f\left(x_{3}\right) \mid X_{2}\right) d \eta_{1} \\
-\int_{Y_{2}} E\left(f\left(x_{1}\right) \mid X_{2}\right) \cdot E\left(f\left(x_{3}\right) \mid Y_{2}\right) d \eta_{2}+\int_{X_{2}} E\left(f\left(x_{1}\right) \mid X_{2}\right) \cdot E\left(f\left(x_{3}\right) \mid X_{2}\right) d \mu_{2}
\end{gathered}
$$

by Lemma 2(i) and the fact that $X_{2}$ is a factor of $Y$ and $Y$ is ergodic. By taking in the latter three summands the conditional expectation with respect to $X_{2}$, we obtain

$$
\begin{gathered}
E(g \otimes g \mid \mathcal{I}) \\
=E\left(E\left(f\left(x_{1}\right) \mid Y_{1}\right) \otimes E\left(f\left(x_{3}\right) \mid Y_{2}\right) \mid \mathcal{I}\right)-\int_{X_{2}} E\left(f\left(x_{1}\right) \mid X_{2}\right) \cdot E\left(f\left(x_{3}\right) \mid X_{2}\right) d \mu_{2} .
\end{gathered}
$$

Using consecutively Lemma 1 and (3), together with Lemma 2(ii), and finally the definition of the relative product, we obtain that

$$
\begin{gathered}
E\left(E\left(f\left(x_{1}\right) \mid Y_{1}\right) \otimes E\left(f\left(x_{3}\right) \mid Y_{2}\right) \mid \mathcal{I}\right) \\
=E\left(E\left(f \otimes f \mid Y_{1} \times_{X_{2}} Y_{2}\right) \mid \mathcal{I}\right)=\int_{X \times X \times X} f\left(x_{1}\right) f\left(x_{3}\right) d \lambda \otimes_{X} \lambda^{*}\left(x_{1}, x_{2}, x_{3}\right) \\
=\int_{X_{2}} E\left(f\left(x_{1}\right) \mid X_{2}\right) \cdot E\left(f\left(x_{3}\right) \mid X_{2}\right) d \mu_{2} .
\end{gathered}
$$

We hence have proved $E(g \otimes g \mid \mathcal{I})=0$ and (44) directly follows.

If $h=h\left(x_{2}\right)$ is in $L^{2}\left(X_{2}\right)$, then $h$ is $Y$-measurable and by (4) we have

$$
\begin{aligned}
& E\left(f\left(x_{1}\right) \cdot h\left(x_{2}\right) \mid Y\right)=h\left(x_{2}\right) \cdot E\left(f\left(x_{1}\right) \mid Y\right) \\
= & h\left(x_{2}\right) \cdot E\left(f\left(x_{1}\right) \mid X_{2}\right)=E\left(f\left(x_{1}\right) \cdot h\left(x_{2}\right) \mid X_{2}\right) .
\end{aligned}
$$

Since the family of the function of the form $f \otimes h$ as above forms a linearly dense subset in $L^{2}(X \times X, \lambda), E(F \mid Y)=E\left(F \mid X_{2}\right)$ for all $F \in L^{2}(X \times X, \lambda)$. Hence $Y=X_{2}$ and the relative Kronecker factor of $\left(X_{1} \times X_{2}, \lambda_{1}\right)$ over $X_{2}$ is trivial. In view of (B), it follows that $\lambda \otimes_{X_{2}} \lambda^{*}$ is ergodic. 
Proof of Theorem 1. At first, assume that $T$ is semisimple. Consider $\lambda_{1}, \lambda_{2} \in$ $J^{e}(T)$. Then, by using Proposition 6.3 from [2], $\lambda_{1} \otimes_{X_{2}} \lambda_{2}^{*}$ is ergodic by semisimplicity of $T$. Therefore $\lambda_{2} \circ \lambda_{1}$ remains ergodic.

If $J_{2}^{e}(T)$ is a subsemigroup, then directly from Proposition 1 it follows that $T$ is semisimple.

Remark 2. The proof of Proposition 1 gives a slightly more general result: Assume that $\lambda$ is an ergodic joining of $S$ (acting on $(Y, \mathcal{C}, \eta)$ ) and $T$ (acting on $(X, \mathcal{B}, \mu)$ ). Then the relative product $\lambda \otimes_{X} \lambda$ is ergodic if and only if the measure $\lambda^{*} \circ \lambda$ on $Y \times Y$ (given by the Markov operator $\Phi_{\lambda}^{*} \circ \Phi_{\lambda}$ on $L^{2}(Y, \eta)$ ) is ergodic. Therefore we obtain an answer to the question by Ryzhikov from [8, p. 95 .

\section{REFERENCES}

[1] H. Furstenberg, Ergodic behaviour of diagonal measures and a theorem of Szemeredy on arithmetic progressions, J. Anal. Math. 31 (1977), 204-256. MR 58:16583

[2] H. Furstenberg, Recurrence in Ergodic Theory and Combinatorial Number Theory, Princeton Univ. Press, 1981. MR 82j:28010

[3] A. del Junco, M. Lemańczyk, M.K. Mentzen, Semisimplicity, joinings and group extensions, Studia Math. 112 (1995), 141-164. MR 97d:28027

[4] A. del Junco, D. Rudolph, On ergodic actions whose ergodic self-joinings are graphs, Erg. Th. Dynam. Syst. 7 (1987), 229-248. MR 89e:28029

[5] M. Lemańczyk, F. Parreau, J.-P. Thouvenot, Gaussian automorphisms whose ergodic selfjoinings are Gaussian, Fund. Math. 164 (2000), 253-293. MR 2001h:37009

[6] M. Lemańczyk, J.-P. Thouvenot, B. Weiss, Relative discrete spectrum and joinings, to appear in Monatshefte Math.

[7] D. Rudolph, An example of a measure-preserving map with minimal self-joinings, and application, J. Anal. Math. 35 (1979), 97-122. MR 81e:28011

[8] V.V. Ryzhikov, Joinings, wreath products, factors and mixing properties of dynamical systems, Russian Acad. Sci. Izv. Math. 42 (1994), 91-114. MR 94h:28016

[9] W.A. Veech, A criterion for a process to be prime, Monatsh. Math. 94 (1982), 335-341. MR 84d:28026

[10] R. Zimmer, Extensions of ergodic group actions, Illinois J. Math. 20 (1976), 373-409. MR 53:13522

Department of Mathematics, Korea Advanced Institute of Science and Technology, TAEJON 305-701, KOREA

E-mail address: ahn@euclid.kaist.ac.kr

Faculty of Mathematics and Computer Science, Nicholas Copernicus University, Chopina 12/18 87-100 Toruń, Poland

E-mail address: mlem@mat.uni.torun.pl 